\section{Case Reports in Ophthalmology}

Case Rep Ophthalmol 2018;9:92-95

DOI: $10.1159 / 000485966$
Published online: January 29, 2018

This article is licensed under the Creative Commons Attribution-NonCommercial 4.0 International License (CC BY-NC) (http://www.karger.com/Services/OpenAccessLicense). Usage and distribution for commercial purposes requires written permission.

\title{
Polypoidal Choroidal Vasculopathy Associated with Optic Disc Coloboma
}

\author{
Yumiko Nakano Akiko Miki Shigeru Honda Makoto Nakamura \\ Department of Surgery, Division of Ophthalmology, Kobe University Graduate School of \\ Medicine, Kobe, Japan
}

\section{Keywords}

Cavitation · Coloboma · Polypoidal choroidal vasculopathy · Photodynamic therapy

\begin{abstract}
Purpose: To report a case of polypoidal choroidal vasculopathy associated with optic disc coloboma. Methods: Case report. Results: A 50-year-old woman presented with optic disc coloboma and retinochoroidal coloboma associated with subretinal hemorrhage and serous retinal detachment (SRD) in her left eye. Optical coherence tomography (OCT) confirmed SRD at the macula and showed a sharply elevated retinal epithelial detachment at the choroidal excavation. OCT also revealed choroidal cavitation along the temporal side of the optic coloboma. Fluorescein angiography showed hyperfluorescent dye leakage and indocyanine green angiography revealed polypoidal lesions. We diagnosed polypoidal choroidal vasculopathy (PCV). PCV was located at the end of the choroidal cavitation. Her left eye was treated with an intraocular injection of the anti-vascular endothelial growth factor aflibercept (2 mg). Photodynamic therapy was performed using the standard protocol 1 week after the intravitreal application of aflibercept. One month after the combined treatment, OCT showed completely resolved SRD and her symptoms disappeared. Her best-corrected visual acuity remained stable and no recurrence was found during a 12-month follow-up period. Conclusion: PCV associated with optic disc coloboma has not been previously reported. The morphological abnormality of choroidal cavitation and choroidal excavation connecting with optic disc coloboma may contribute to the development of PCV in this case.
\end{abstract}




\section{Case Reports in Ophthalmology}

Ocular coloboma is a congenital disease and a leading cause of pediatric visual impairment. The underlying etiology is considered to be the failure of optic fissure closure during embryogenesis. The defect can occur in the iris, retina, choroid, and/or optic nerve; major complications include cataract and retinal detachment. Choroidal neovascularization (CNV) is a complication of retinochoroidal and optic disc colobomas [1]; however, colobomarelated polypoidal choroidal neovascularization (PCV) has not been reported. Here we report a case of PCV associated with optic disc coloboma.

A 50-year-old woman had experienced left-sided anorthopia for 1 year. Because her symptoms worsened, she visited an eye clinic; pit-macular syndrome was suspected and she was referred to our hospital. At her first visit to our hospital, her best-corrected visual acuity in the affected eye was 20/16 and that in her right eye was 20/20. Fundus examination of her left eye revealed optic disc and retinochoroidal colobomas associated with subretinal hemorrhage around the optic disc (Fig. 1a, b) and macular serous retinal detachment (SRD). Optical coherence tomography (OCT) confirmed macular SRD (Fig. 1c-e) and a sharply elevated retinal pigment epithelial detachment connecting with "the double-layer sign," which represents a branched vascular membrane above the retinal pigment epithelium (RPE) (Fig. 1c). OCT also revealed a peripapillary choroidal cavitation (PCC) at the temporal margin of the optic disc coloboma (Fig. 1d, e). Fluorescein angiography showed hyperfluorescent dye leakage, and indocyanine green angiography revealed polypoidal lesions (Fig. 1f, g); PCV was diagnosed. Her left eye was treated with an intraocular injection of antivascular endothelial growth factor (VEGF) (2 mg aflibercept). Photodynamic therapy (PDT) was performed (standard protocol) 1 week after the injection of aflibercept. One month later, OCT revealed completely resolved SRD (Fig. 2b, c). The best-corrected visual acuity of the affected eye remained stable, and no recurrence was found during a 12-month follow-up period.

The pathogenesis of $\mathrm{CNV}$ in retinochoroidal colobomas considered that the abrupt termination and abnormal architecture of RPE and the Bruch's membrane at the margin may predispose these patients to the development of subretinal neovascularization. In 2003, Guirgis et al. [2] reported a case of optic disc coloboma accompanied by CNV at the margin of the coloboma. There is a report about the association between optic disc coloboma and PCC [3]. In their study, PCC was found at the margin of choroidal coloboma. They speculated that the morphologic changes at the border of colobomas and PCC might be different stages of the same disorder. Here the location of PCV was at the end of PCC in the choroidal excavation. PCV can occur in association with choroidal excavation. It is suggested that mechanical stress owing to abnormal choroidal morphology accompanied with choroidal excavation and PCC might induce production of vascular endothelium growth factor (VEGF), contributing to the development of PCV.

There are two reports on CNV associated with retinochoroidal and optic disc coloboma $[4,5]$. Goodwin et al. [4] reported the efficacy of anti-VEGF treatment with $0.5 \mathrm{mg}$ of ranibizumab injected into the vitreous. An intravitreal injection of anti-VEGF (bevacizumab) with reduced fluorescence PDT was also reported to be effective [5]. There are several options for the management of PCV associated with coloboma. Here thermal laser treatment was not appropriate because the scarring may lead to severe loss of vision. PDT monotherapy or transpupillary thermotherapy may cause significant loss of vision because of damage to the RPE and the inner choroid. A recent study by Koh et al. [6] showed the efficacy of PCV treatment using intravitreal injections of anti-VEGF (ranibizumab) combined with PDT (standard protocol). We treated the patient with an intravitreal injection of anti-VEGF (aflibercept) combined with PDT (standard protocol). The effect of this first treatment has been maintained for $>1$ year. No progression of RPE atrophy was detected on the follow-up. 
This is the first report describing PCV associated with optic disc coloboma. The combination of intravitreal injection of anti-VEGF agent and PDT was effective in this case.

\section{Statement of Ethics}

Written informed consent was obtained from the patient.

\section{Disclosure Statement}

The authors have no proprietary or commercial interest in any materials discussed in this article. No conflicting relationship exists for any author.

\section{References}

1 Onwochei BC, Simon JW, Bateman JB, Couture KC, Mir E: Ocular colobomata. Surv Ophthalmol 2000;45:175-794.

2 Guirgis MF, Lueder GT: Choroidal neovascular membrane associated with optic nerve coloboma in a patient with CHARGE association. Am J Ophthalmol 2003;135:919-920.

-3 Holak HM, Kohlhase J, Holak SA, Holak NH: New recognized ophthalmic morphologic anomalies in CHARGE syndrome caused by the R2319C mutation in the CHD7 gene. Ophthalmic Genet 2008;29:7984.

4 Goodwin P, Shields CL, Ramasubramanian A, Brown GC, Shields JA: Ranibizumab for coloboma-related choroidal neovascular membrane in a child. J AAPOS 2009;13:616-617.

5 Bhende M, Suganeswari G, Gopal L, Bhende PS, Gopal L, Rao C: Choroidal neovascularization associated with coloboma of the choroid: a series of three cases. Ind J Ophthalmol 2011;59:148-151.

-6 Koh A, Lee WK, Chen LJ, Chen SJ, Hashad Y, Kim H, Lai TY, Pilz S, Ruamviboonsuk P, Tokaji E, Weisberger A, Lim TH; EVEREST study: Efficacy and safety of verteporfin photodynamic therapy in combination with ranibizumab or alone versus ranibizumab monotherapy in patients with symptomatic macular polypoidal choroidal vasculopathy. Retina 2012;32:1453-1464.

This study was presented at the 120th Annual Meeting of the Japanese Ophthalmological Society in Sendai, Japan on April 7, 2016. 


\section{Case Reports in Ophthalmology}
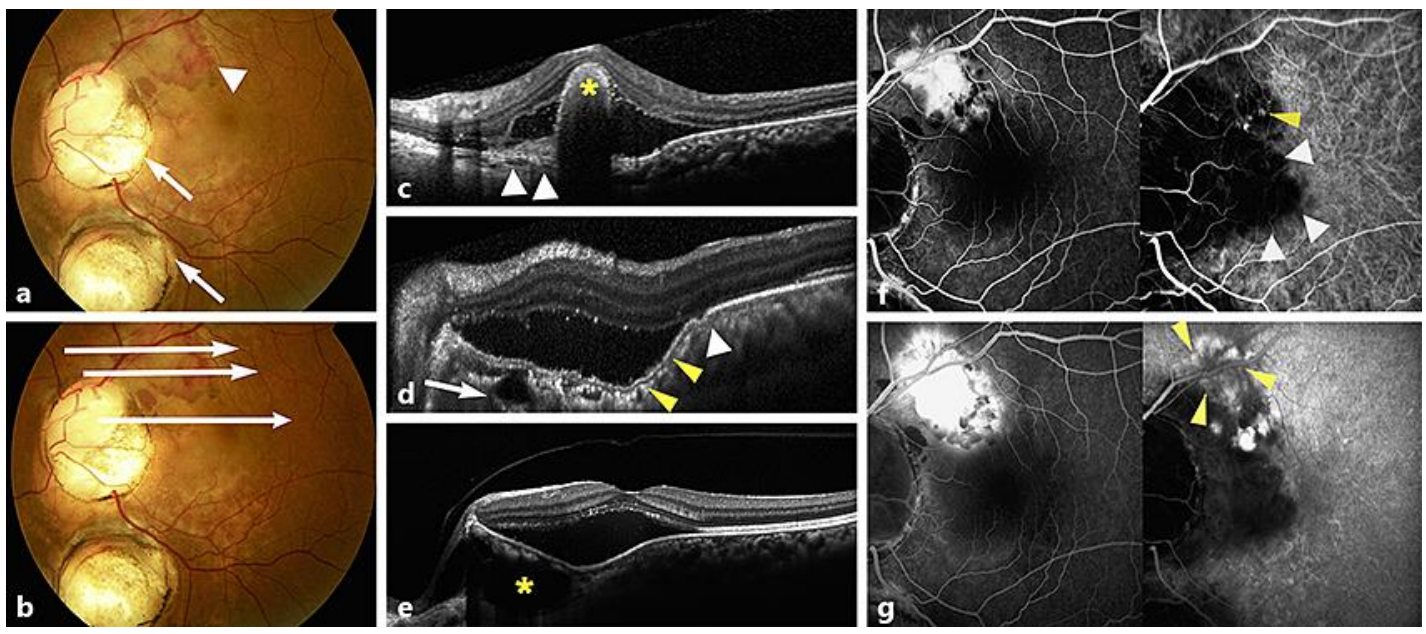

Fig. 1. a Color fundus photograph shows optic disc and retinochoroidal coloboma (white arrow) and subretinal hemorrhage (white arrowhead) c-e The horizontal SD-OCT section through the indicated lesion (b, white arrow) was shown in order. c The SD-OCT shows elevated pigment epithelium detachment (yellow asterisk) and "double-layer sign" (white arrowheads) accompanied with serous detachment. $\mathbf{d}$ The SD-OCT shows choroidal excavation (yellow arrowheads) and intrachoroidal cavitation (white arrow) with serous detachment. There is an abnormality in the retinal pigment epithelium (white arrowhead) corresponding to point dye leakages on indocyanine green angiography (ICGA) (f, right panel, yellow arrowheads). e The SD-OCT shows intrachoroidal cavitation (yellow asterisk) with serous detachment. $\mathbf{f}, \mathbf{g}$ Middle phase (f, left panel) and late phase ( $g$, left panel) fluorescein angiography shows dye leakage from PCV. Middle phase (f, right panel) and late phase (g, right panel) ICGA shows a polypoidal lesion (yellow arrow heads). Hyporefractive lesion of ICGA (white arrowheads) represents the area of choroidal excavation
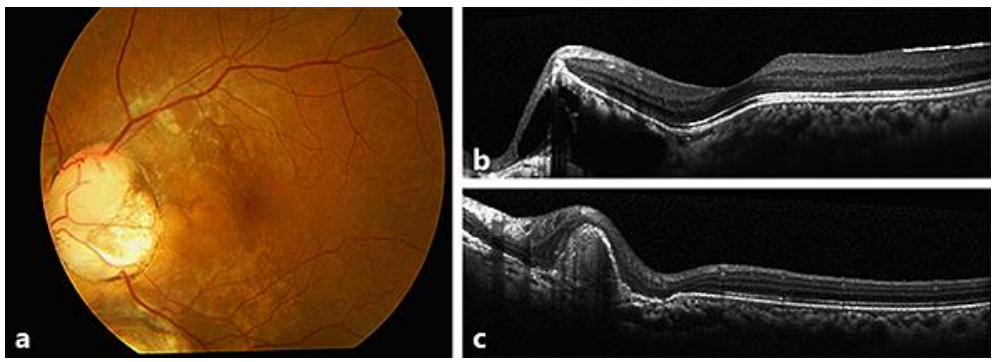

Fig. 2. a Color fundus photograph shows the regression of subretinal hemorrhage 1 year after treatment. b, c The SD-OCT shows the resolution of subretinal fluid at the macula and the PCV lesion. 